\title{
Responses to Drought and Salinity in the Endangered Species Ligularia sibirica (L.) Cass.
}

\author{
Andreea N. MATEI ${ }^{1,2}$, Mohamad AL HASSAN ${ }^{2}$, Monica BOSCAIU ${ }^{3}$, Valeriu ALEXIU ${ }^{1}$, Oscar VICENTE ${ }^{*}$ \\ ${ }^{1}$ Faculty of Science, University of Pitesti, Romania \\ ${ }^{2}$ Instituto de Biología Molecular y Celular de Plantas (IBMCP, UPV-CSIC) and ${ }^{3}$ Instituto Agroforestal \\ Mediterráneo (IAM, UPV), Universitat Politècnica de València, Spain \\ *)Corresponding author, e-mail: ovicente@ibmcp.upv.es
}

BulletinUASVM Horticulture 73(2) / 2016

Print ISSN 1843-5254, Electronic ISSN 1843-5394

DOI:10.15835/buasvmcn-hort:12286

\begin{abstract}
The negative effects of environmental stress factors on plant distribution and survival are increasing due to climate change and anthropogenic activities. We have analysed some responses to abiotic stress in Ligularia sibirica, a postglacial relict that is critically endangered in Europe. L. sibirica seedlings were subjected to water or salt stress treatments in the greenhouse. After the treatments, plant material was harvested and several growth parameters were measured; leaf contents of common osmolytes, the degree of oxidative stress affecting the plants and the level of antioxidant phenolic compounds were also determined. Both, drought and, especially, salt stress had a negative effect on the growth of $L$. sibirica plants. Treated plants showed an increase in proline (Pro) and total soluble sugars (TSS) levels, stronger under salt stress. Malondialdehyde (MDA, an oxidative stress biomarker) contents almost doubled, and antioxidant phenolics increased significantly in salt-stressed, but not in water-stressed plants. Pro accumulation can be used as a salt and drought stress biomarker in L. sibirica and, together with TSS, likely contributes to osmotic adjustment under stress. Increase of antioxidant phenolics appears to partly compensate the salt-induced generation of oxidative stress.
\end{abstract}

Keywords: antioxidants; drought; Ligularia sibirica; osmolytes; salinity

\section{Introduction}

Ligularia sibirica is a postglacial relict that is strongly affected by climate change and human activities, to the extent that it is regarded as a critically endangered species in Europe (Witkowski, 2003). Apart from biodiversity issues, the species has some economic interest, as an ornamental and medicinal plant. To our knowledge, $L$. sibirica has not been previously investigated regarding its responses to abiotic stress conditions.

\section{Aims and objectives}

The aim of this study was to analyse some physiological and biochemical responses of $L$. sibirica to controlled drought and salt stress treatments, to obtain insights on its stress response mechanisms, which could help to design strategies for the conservation and practical use of the species.

\section{Materials and methods}

L. sibirica seeds were sown on a moistened mixture of peat (50\%), perlite (25\%) and vermiculite (25\%), and grown in controlled greenhouse conditions, using Hoagland nutritive solution for watering. Two-month old plants were subjected to four-weeks stress treatments, applied by completely ceasing irrigation (drought stress) or by adding $\mathrm{NaCl}$ to the Hoagland solution to a final concentration of $200 \mathrm{mM}$ (salt stress). After harvesting, growth parameters (fresh weight, stem length and leaf length) were measured. Leaf contents of Pro and TSS (common osmolytes), MDA, total phenolic compounds (TPC), and total flavonoids (TF) were determined using standard spectrophotometric assays. The statistical analysis was carried out using the Statgraphics Centurion XVI programme. 
Tab. 1. Stress-induced growth inhibition in Ligularia sibirica. Growth parameters: fresh weight (FW), stem length (LS), and leaf length (LL), in control and in drought and salt stressed plants, after onemonth treatments.

\begin{tabular}{cccc}
\hline Treatments & FW $(\mathrm{g})$ & SL $(\mathrm{cm})$ & LL $(\mathrm{cm})$ \\
\hline Control & $4.10 \pm 0.59$ & $4.12 \pm 0.26$ & $1.75 \pm 0.11$ \\
\hline Water Stress & $1.47 \pm 0.21$ & $2.80 \pm 0.29$ & $1.47 \pm 0.12$ \\
\hline $200 \mathrm{mM} \mathrm{NaCl}$ & $1.14 \pm 0.18$ & $1.42 \pm 0.30$ & $1.00 \pm 0.13$ \\
\hline
\end{tabular}

Tab. 2. Relative increase in leaf levels of proline (Pro), total soluble sugars (TSS), malondialdehyde (MDA), total phenolic compounds (TPC) and total flavonoids (TF), in Ligularia sibirica plants, after one-month water and salt stress treatments, as compared to non-stressed control plants

\begin{tabular}{cccccc}
\hline Treatments & $\begin{array}{c}\text { Pro } \\
\text { (fold-) }\end{array}$ & $\begin{array}{c}\text { TSS } \\
\text { (fold-) }\end{array}$ & $\begin{array}{c}\text { MDA } \\
\text { (fold-) }\end{array}$ & $\begin{array}{c}\text { TPC } \\
\text { (fold-) }\end{array}$ & $\begin{array}{c}\text { TF } \\
\text { (fold-) }\end{array}$ \\
\hline Control & 1.00 & 1.00 & 1.00 & 1.00 & 1.00 \\
\hline Water Stress & $2.65 \pm 0.40$ & $1.59 \pm \mathbf{0 . 0 9}$ & $1.19 \pm 0.06$ & $1.06 \pm 0.17$ & $1.01 \pm 0.12$ \\
\hline $200 \mathrm{mM} \mathrm{NaCl}$ & $11.62 \pm 1.90$ & $1.97 \pm 0.06$ & $1.92 \pm 0.16$ & $1.48 \pm 0.15$ & $1.43 \pm 0.10$ \\
\hline
\end{tabular}

\section{RESULTS AND DISCUSSION}

Both drought and salinity had a strong negative effect on the growth of $L$. sibirica plants, but more severe in the case of salt stress, as shown by the reduction in fresh weight, and in stem and leaf lengths (Table 1).

The stress treatments induced a relative increase in the leaf levels of common osmolytes, such as proline and, to a lesser extent, total soluble sugars; here again, accumulation of these compatible solutes was higher in salt-stressed than in water-stressed plants (Table 2). Even though soluble sugars contents increase less than two-fold under stress, their absolute values in control plants were quite high (ca. $100 \mathrm{mg}$ eq. glucose per g DW). Therefore, proline and soluble sugars are most likely involved in the maintenance of osmotic balance, protecting the plants against drought- and salinity-induced cellular dehydration - without ruling out the possible contribution of additional, not yet tested osmolytes.

Under our experimental conditions, salt stress (but not drought) generated oxidative stress in the plants, as revealed by the increase in MDA levels (Table 2). One general response to different abiotic stresses is based on the activation of antioxidant systems, both enzymatic and non-enzymatic. We have indeed detected the increase in the levels of total phenolics and total flavonoids (Table 2), plant secondary metabolites which include many strong antioxidant compounds.

\section{Conclusion}

The work presented here provides a first insight into the mechanisms of response to drought and soil salinity of Ligularia sibirica, a critically endangered species in Europe. Both stresses inhibited growth, although the plants appeared to be more sensitive to salt than to water stress. Proline accumulation can be used as an appropriate stress biomarker in L. sibirica and, together with soluble sugars, likely contributes to osmotic adjustment under stress conditions. Increase of antioxidant phenolics appears to partly compensate the salt-induced generation of oxidative stress in the plants.

Acknowledgements. A.N.M. is indebted to the Erasmus+ Placement programme for funding her internship at the IBMCP lab, as a part of her PhD studies.

\section{REFERENCES}

1. Witkowski ZJ, Król W, Solarz W, Kukuła K, Okarma H, Pawłowski J, Perzanowski K, Ruzicka T, Sandor J, Stanova V, Tasenkevich L, Vlasin M (2003). Carpathian List of Endangered Species, Edit. Carpathian Ecoregion Initiative, Vienna, Austria and Krakow, Poland: pp. 29-33. 